\title{
Nano-crystals from Ionic Liquids: Formation and Characterization Using Atomic Force Microscopy
}

\author{
*J. J. Nainaparampil, J. H. Sanders, K. C. Eapen, and J. S. Zabinski \\ Air Force Research Laboratory, Wright-Patterson Air Force Base, Dayton, Oh. 45433
}

The structure of ionic liquid in the liquid phase is almost unknown at the present time. During recent years there have been reports about their fundamental structure, which was determined using both simulations and experiments. We report the micro-nano crystallization of ionic liquids 1,2-Dimethyl-3-butyl imidazolium hexafluorophosphate (DMBI-PF 6 ), 1-Butyl-3-methyl imidazolium-2-(2-methoxyethoxy)ethyl sulfate ( BMIMEE sulfate ) and DMBI-Cl. It has been observed that macro crystals can show different behavior and structures than nanocrystals. The DMBI-PF6 is applied as a thin film while BMI-MEE sulfate and DMBI-Cl structures are created using Dip Pen Nanolithography (DPN), which is a technique for using an AFM tip as a nano "ink pen" [1]. The DMBIPF6 films form micro droplets in ambient conditions but undergo drastic changes including formation of nanocrystals when agitated or touched with a biased or unbiased AFM tip. Crystallization of DMBI-PF 6 from the liquid phase with tip bias is shown to be a reversible process based on tip polarity. The nanotribological characteristics of the structures are studied, including mobility, diffusion, adhesion and scratch resistance.

Figure 1(a) shows the phase image of a line drawn with DMBI-Cl on hydrogenated silicon surface with a positively biased tip. It was observed that material transferred to the tip such that "tip convolution" effects determined topography. An atomic force microscopy (AFM) image of a surface is basically a convolution of tip geometry and surface structures [2]. The droplet-like structures observed in Figure 1(a) appear to diffuse away from the centerline and after 12 minutes, the separation has slightly increased (Figure 1(b). The separation of droplets over time suggests charging effects are active. This phenomenon is discussed in detail in the presentation.

Surface chemistry and structure are known to affect the crystallization process [3] and hence hydrogenated and oxidized surfaces were investigated. The crystalline structure of the BMI-MEE sulfate lines drawn using DPN indeed depended on both bias and surface preparation. Figure 2 shows the nanolithographed patterns obtained for BMI-MEE sulfate. Fig 2(a) shows the patterns obtained for hydrogenated silicon surface. The circular head structure is formed by diffusion material while the tip was stationary. Upon moving the tip, the width of the path changes depending on the speed of the tip and height of the tip from the surface. Fig 2(b) shows the patterns obtained for BMI-MEE sulfate lines drawn on a thermally oxidized silicon surface. The significant change in the nanocrystal shape is due to the difference in surface chemistry and structure compared to the hydrogenated surface. This is a clear indication of the effect of interfacial forces on nanoscale crystal formation [4].

AFM studies of nano structures formed by DPN can help understand their important nano-tribological characteristics when they are applied as lubricants. 


\section{References}

[1] Piner, R. D.; Jin, Z.; Feng, X.; Hong, S.; Mirkin, C. A. Science 283 (1999) 661.

[2] J. Vesenka, T. Marsh, R. Miller, and E. Henderson, J. Vac. Sci. \& Technol. B 14 (1996) 1413.

[3] Lin J. S., et.al. Langmuir, 14 (1998) 4843

[4] Birdi K. S., Self-Assembly Monolayer (SAM) Structures, Plenum Press, New York, 1999.

[5] This work is supported by AFOSR and Materials Directorate at Wright Patterson AFB

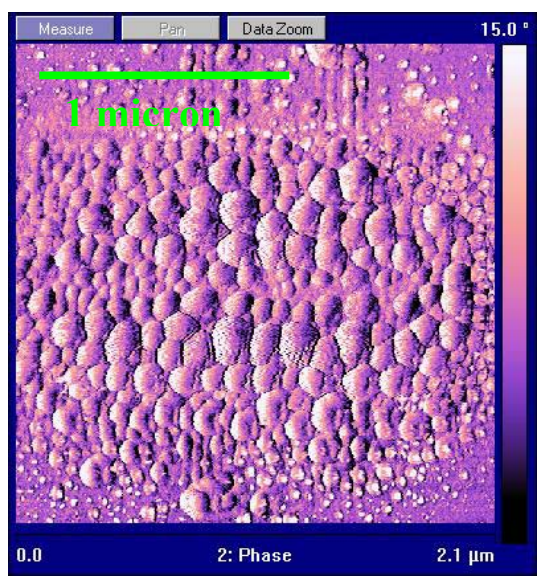

(a)

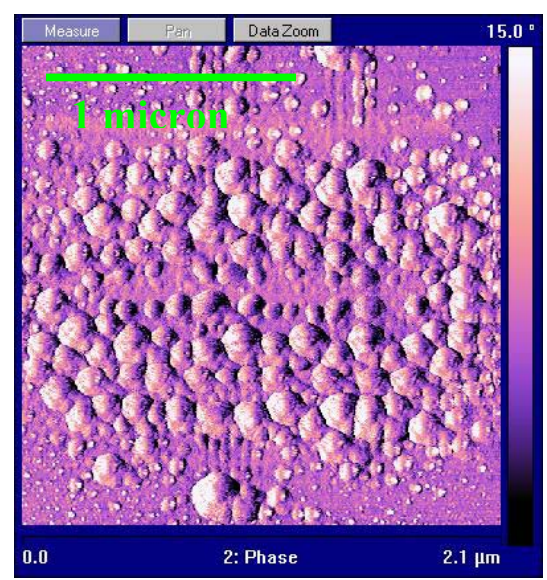

(b)

Figure 1(a) Line of DMBI-CL formed on hydrogenated silicon using DPN (b) structures separating over 12 minutes.

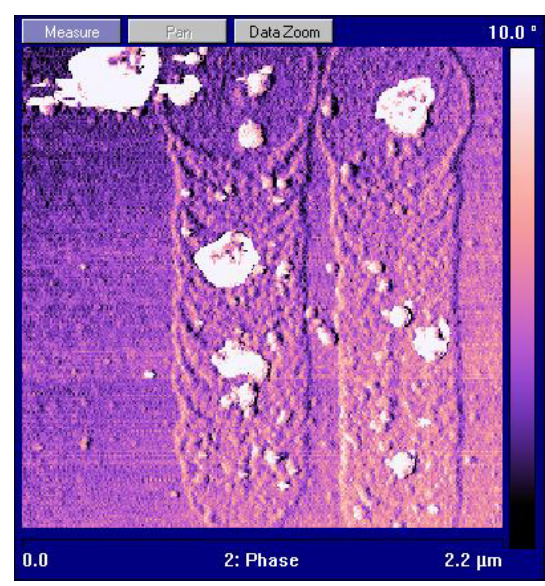

(a)

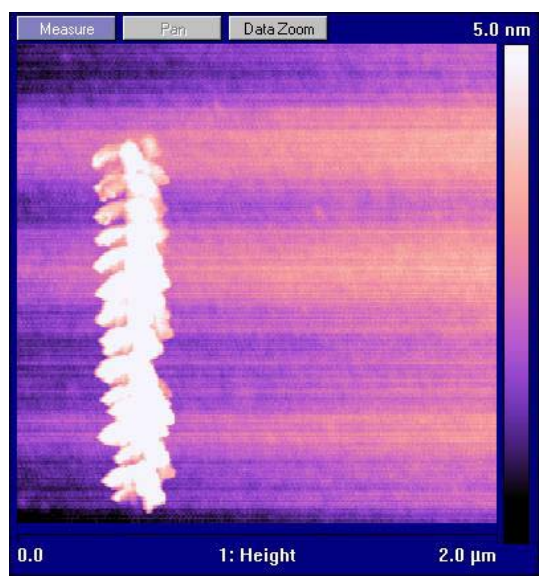

(b)

Figure 2(a) Pattern obtained by Dip Pen Lithography of BMI-MEE sulfate on hydrogenated silicon with a $-5 \mathrm{~V}$ biased tip. (b) Nano crystal growth observed for the same ionic liquid on a thermally oxidized silicon surface. 